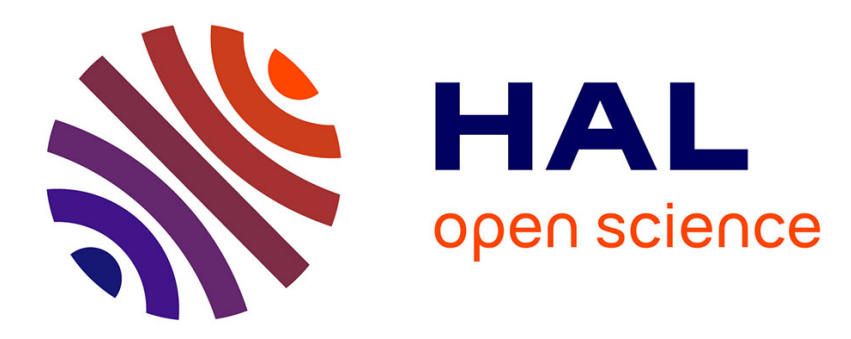

\title{
Le vieillissement du système immunitaire: du fondamental à la clinique
}

\author{
H. Vallet, T. Fali, D. Sauce
}

\section{To cite this version:}

H. Vallet, T. Fali, D. Sauce. Le vieillissement du système immunitaire: du fondamental à la clinique. La Revue de Médecine Interne, 2019, 40 (2), pp.105-111. 10.1016/j.revmed.2018.07.005 hal02352200

\section{HAL Id: hal-02352200 \\ https://hal.sorbonne-universite.fr/hal-02352200}

Submitted on 6 Nov 2019

HAL is a multi-disciplinary open access archive for the deposit and dissemination of scientific research documents, whether they are published or not. The documents may come from teaching and research institutions in France or abroad, or from public or private research centers.
L'archive ouverte pluridisciplinaire $\mathbf{H A L}$, est destinée au dépôt et à la diffusion de documents scientifiques de niveau recherche, publiés ou non, émanant des établissements d'enseignement et de recherche français ou étrangers, des laboratoires publics ou privés. 


\title{
Le vieillissement du système immunitaire : du fondamental à la clinique Aging of the immune system: from fundamental to clinical data
}

\author{
H. Vallet ${ }^{1,2,3}$, T. Fali $^{1,3}$, V. Appay ${ }^{1,3}$, D. Sauce ${ }^{1,3^{*}}$
}

1. INSERM U1135, Centre d'Immunologie et des Maladies Infectieuses (CIMI-Paris), Paris, France.

2. Hôpital Pitié-Salpêtrière, Unité Péri-Opératoire Gériatrique (UPOG), Département Gériatrie, Paris, France.

3. Sorbonne Université, Université Pierre et Marie Curie, Paris, France

*Auteur correspondant:

Delphine Sauce, Centre d'Immunologie et des Maladies Infectieuses (Cimi-Paris), INSERM

U1135, Sorbonne Universités, Hôpital Pitié-Salpêtrière, 75013 Paris, France.

Fax: $+33-1-40-77-97-34$.

Téléphone: +33-1-40-77-98-89.

Email: delphine.sauce@upmc.fr 


\title{
Le vieillissement du système immunitaire : du fondamental à la clinique Aging of the immune system: from fundamental to clinical data
}

\author{
H. Vallet ${ }^{1,2,3}$, T. Fali ${ }^{1,3}$, D. Sauce ${ }^{1,3^{*}}$ \\ 1. INSERM U1135, Centre d'Immunologie et des Maladies Infectieuses (CIMI-Paris), Paris, \\ France. \\ 2. Hôpital Pitié-Salpêtrière, Unité Péri-Opératoire Gériatrique (UPOG), Département Gériatrie, \\ Paris, France. \\ 3. Sorbonne Universités, Université Pierre et Marie Curie, Paris, France \\ *Auteur correspondant: \\ Delphine Sauce, Centre d'Immunologie et des Maladies Infectieuses (Cimi-Paris), INSERM \\ U1135, Sorbonne Universités, Hôpital Pitié-Salpêtrière, 75013 Paris, France. \\ Fax: +33-1-40-77-97-34. \\ Téléphone: +33-1-40-77-98-89. \\ Email: delphine.sauce@upmc.fr
}




\section{Résumé :}

L'espérance de vie ne cesse d'augmenter en raison de progrès importants dans la prévention, le retard ou la guérison de diverses pathologies normalement rencontrées lors du vieillissement. Cependant, les progrès scientifiques et médicaux sont encore nécessaires pour comprendre la cause sous-jacente de l'occurrence disparate des comorbidités avec le vieillissement. D'une part, le vieillissement altère profondément le système immunitaire; il est caractérisé par de nombreux changements de l'hématopoïèse, des systèmes adaptatifs et innés, associés à un environnement pro-inflammatoire. D'autre part, les événements stressants (aigus ou chroniques) peuvent également avoir un impact sur le système immunitaire par la sécrétion d'hormones, qui sont également altérées par le vieillissement. Le domaine de la psychoneuroimmunologie fournit maintenant des preuves que, dans les conditions médicales aiguës, les personnes âgées ne sont pas égales dans leurs réponses aux facteurs de stress. Des paramètres (extrinsèques et intrinsèques) pourraient interférer avec la capacité des personnes âgées à monter une réponse immunitaire efficace. L'objectif de cette revue est de fournir un aperçu de la littérature (des observations fondamentales aux observations cliniques) pour établir un panorama global de l'immunosénescence. Comprendre ce phénomène pourrait nous permettre de cibler des voies fondamentales liées à l'âge et ainsi ouvrir de nouvelles perspectives pour améliorer la durée de vie mais aussi pour se maintenir en bonne santé plus longtemps.

Mots-clé : vieillissement, inflammation, stress, immunité 


\begin{abstract}
Major progress in preventing, delaying or curing various pathologies normally encountered in old age results in a continuous improvement in life expectancy. However, understanding the underlying cause of the disparate comorbidities occurrence with aging remains a priority in order to propose adapted care for the older population. In one hand, aging profoundly impairs the immune system; it is characterized by many changes in haematopoiesis, adaptive and innate systems, and is associated with pro-inflammatory environment. In another hand, stressful events (acute or chronic) can also impact the immune system through the secretion of hormones, which are also altered with aging. Blooming evidences from psychoneuroimmunology field suggest that, in acute medical conditions, elderly people are not equal in their responses to stressors depending on many extrinsic and intrinsic parameters. These factors could interfere with elderly's ability to mount an effective immune response.
\end{abstract}

The objective of this review is to provide an overview of the literature (from fundamental to clinical observations) to draw a global picture of immunosenescence. Understanding this process could enable us to target fundamental age-related pathways and thus open new avenues in improving both lifespan and health span.

Keywords: aging, inflammation, stress, immunity 
La population mondiale vieillie, notamment dans les pays industrialisés. La population âgée

de plus de 60 ans a triplé au cours des 50 dernières années et triplera encore à l'horizon 2050. D'après les estimations, $10 \%$ de la population européenne aura plus de 80 ans en 2050 et les centenaires seront 3.2 millions à travers le monde, soit 18 fois plus qu'en 2000 [1]. Cette évolution, liée à la diminution de la mortalité et de la natalité, pose de réelles questions sociétales, économiques, médicales, scientifiques et éthiques. Une meilleure compréhension des phénomènes et mécanismes à l'origine du vieillissement est primordiale pour améliorer la prise en charge médicale de nos aînés et réduire les coûts pour la société. Le but n'est pas nécessairement d'allonger à tout prix la durée de la vie mais sa qualité. En effet, un vieillissement « réussi » qui permet de gagner en espérance de vie sans incapacité est bénéfique tant pour l'individu que pour la société.

Le vieillissement ne peut se réduire à être défini par un âge civil. II s'agit d'un phénomène complexe associant des modifications physiologiques et des pathologies chroniques. Ces deux éléments entrainent une diminution des capacités de réserve de l'individu qui se traduit par une perte d'autonomie, de qualité de vie et qui parfois mène au décès du patient [2]. La capacité d'une personne âgée à surmonter un évènement médical aigu (ou stress aigu) dépend de l'importance de ses réserves fonctionnelles au moins autant que de l'intensité du stress subi. Cette capacité est également appelée capacité de résilience.

La particularité du phénomène de vieillissement est son hétérogénéité. II existe de nombreuses variabilités intra et inter-individuelles. En effet, tous les organes ne vieillissent pas de la même manière au sein d'un individu. De même, tous les individus ne sont pas égaux face au vieillissement. L'origine de cette variabilité n'est pas intégralement comprise mais il existe de nombreux facteurs environnementaux, génétiques et biologiques entrant en ligne de compte $[3,4]$.

Parmi les organes et systèmes impactés par le vieillissement, le système immunitaire subit de larges modifications. Alors que les connaissances dans le domaine de l'immunosénescence vont croissantes depuis plusieurs années, l'impact du stress sur le système immunitaire du patient âgé est encore mal connu. Hors une meilleure connaissance 
dans ce domaine pourrait permettre d'envisager des thérapeutiques ciblées afin d'améliorer le pronostic des patients âgés.

L'objectif de cette revue est de faire un état des lieux des connaissances concernant le vieillissement du système immunitaire, des liens existant avec le stress et d'entrevoir des stratégies expérimentales pour limiter ce phénomène.

\section{Modifications du système immunitaire au cours du temps}

Le vieillissement est associé à un déclin des compétences du système immunitaire, appelé immunosénescence, touchant aussi bien le compartiment inné qu'adaptatif [5]. II s'y associe un deuxième phénomène appelé « inflamm-aging » caractérisé par une production accrue de cytokines pro-inflammatoires. L'ensemble de ces modifications diminue les capacités du patient âgé à produire une réponse immune efficace contre les antigènes étrangers (Figure 1).

\subsection{Modifications du système immunitaire inné}

Le vieillissement impacte peu les cellules du système immunitaire inné en termes d'effectif. En revanche, il entraine de profondes modifications fonctionnelles.

Ainsi le nombre de monocytes et de macrophages d'un sujet âgé est relativement comparable à celui d'un sujet jeune. On assiste en revanche à une évolution des monocytes vers un profil plus inflammatoire. Ainsi, le nombre de monocytes pro-inflammatoires (CD14high/CD16+) et non conventionnels (CD14low/CD16+) augmente avec l'âge, au détriment des monocytes conventionnels (CD14+/CD16-) [6]. De plus, les monocytes des patients âgés expriment plus fortement le CD11b (intégrine impliquée dans la migration cellulaire) et plus faiblement la L-selectine (molécule impliquée dans l'adhésion épithéliale) ce qui affecte la fonctionnalité de ces monocytes [7,8]. Enfin, les monocytes des patients âgés présentent une diminution de l'expression de TLR1 à leur surface [6] mais une augmentation de la capacité de production de TNF- $\alpha$ via la stimulation de TLR4 [7]. 
Les neutrophiles sont également impactés par le vieillissement. Alors que leur nombre reste relativement constant au cours du temps [9], leurs fonctionnalités sont altérées [10,11]. Ainsi, on assiste à des modifications phénotypiques vers un profil de neutrophiles plus suppressifs (CD16bright/CD62Ldim) [12]. De plus, leur capacité d'activation après stimulation par le LPS est plus faible, leurs capacités de phagocytose, de chimiotactisme et de production de résidu du stress oxydatif sont diminuées [12-14]. Ces modifications fonctionnelles sont le reflet de modifications au sein des voies de signalisation du neutrophile. II existe par exemple des altérations de la transduction de signal via les «Toll-like recepteurs (TLRs) », le « granulocyte-macrophage colony stimulationg factor» (GM-CSF) ou encore la voie «p38 mitogen-activated protein kinase » (MAPK) [9].

\subsection{Les modifications du système adaptatif}

\section{Lymphocytes $T$}

Le vieillissement du compartiment lymphocytaire $\mathrm{T}$ est caractérisé par 4 grands types de modifications, illustrés en Figure 1 :

a) Une diminution du nombre de lymphocytes $T$ (LT) naïfs, secondaire à l'involution thymique. Le thymus, organe lymphoïde primaire, est le siège de l'évolution des précurseurs lymphoïdes en LT naïfs. Au cours du temps, il subit de profondes modifications fonctionnelles. La masse de tissu fonctionnel diminue au profit d'une accumulation graisseuse entrainant une diminution de la production de LT naïfs avec l'âge $[15,16]$.

b) Un appauvrissement du répertoire T par restriction du TCR. Le TCR, par phénomène de réarrangements géniques, a la capacité de reconnaitre une infinité d'antigènes. Or, au cours du vieillissement, le taux de réarrangements diminue entrainant une perte de diversité du compartiment $\mathrm{T}$ ce qui conduit à une moins bonne capacité à reconnaitre un nouvel antigène $[17,18]$.

c) Une augmentation de la proportion de LT mémoires oligoclonaux, particulièrement impliqués dans le contrôle d'infections virales persistantes telles que le cytomégalovirus [19]. 
d) Un raccourcissement des télomères

On note également, au cours du vieillissement, des modifications du profil d'expression génique au sein des sous-populations lymphocytaires $T$ touchant aussi bien des marqueurs de surface que des récepteurs aux chimiokines/cytokines, des molécules effectrices ou des facteurs de transcription. Certains LT mémoires modifient leur phénotype : ils perdent l'expression du CD28, acquièrent des marqueurs de cellules NK (CD57+, CD16+), voir réexpriment le CD45RA qui est habituellement un marqueur des LT naïfs $[20,21]$. Ces cellules T effectrices/sénescentes ont de faibles capacités de prolifération et de production d' IL-2 [21]. De nombreuses modifications ont été retrouvées au sein de ces cellules CD28- tel qu'une augmentation de l'expression de CX3CR1, une augmentation de l'expression d'IL-13, CCL4 et Granzyme B, une diminution de l'expression de I'IL-7R et de l'IL-12R [22]. On retrouve également une augmentation de l'expression des facteurs de transcription T-bet (impliqué dans le développement TH1) et EOMES (impliqué dans la production d'IFN $\gamma$, de perforine et de Granzyme B). En revanche l'expression de MYC, impliqué dans l'apoptose et la différenciation, est réduite chez la personne âgée [22].

Pour finir, le taux LT régulateurs CD4+ FOXP3+ augmente avec l'âge [23] et leur capacité à réguler les LT CD4+ par l'IL-10 est plus importante [24].

\section{Lymphocytes B}

II a été rapporté que les changements associés à l'âge dans la distribution des cellules B périphériques reflètent à la fois une diminution de la production de cellules $B$ à partir de la moelle osseuse et une augmentation de la longévité des lymphocytes B. Effectivement, le nombre de cellules $B$ dans la périphérie diminue chez les personnes âgées. Dû à la diminution de la génération des cellules $B$ progénitrices précoces, la production de nouvelles cellules B naïves est réduite $[25,26]$ et, par conséquent, les cellules B mémoire ayant rencontré un l'antigène sont majoritaires [26]. En outre, la recombinaison de commutation de classe est altérée dans les cellules B mémoire ce qui conduit à une production altérée d'anticorps protecteurs de plus haute affinité, participant ainsi au déclin de la qualité de la 
réponse humorale avec le vieillissement. En outre, l'expansion de cellules B mémoires oligoclonales est reliée à des incidences accrues de malignité des lymphocytes B [27] ou de maladies auto-immunes observées chez les personnes âgées.

\subsection{Inflamm-aging}

Le vieillissement s'accompagne d'un état inflammatoire systémique chronique de bas grade appelé « inflamm-aging » [28]. II se caractérise par la production accrue de cytokines proinflammatoires telles que I'IL-1, I'IL-6, le «tumor necrosis factor alpha » (TNF- $\alpha$ ) ainsi que par la production de C-réactive protéine (CRP) [29,30]. Le niveau de cet état proinflammatoire est associé à un plus mauvais pronostic chez le patient âgé : augmentation de la morbi-mortalité, sarcopénie et fragilité [30].

L'origine de cet état pro-inflammatoire n'est pas complètement comprise à l'heure actuelle. Cette complexité tient au fait que de nombreux tissus et organes participent à la production de stimuli inflammatoires. Ceci inclut le système immunitaire mais aussi le tissu adipeux, le muscle squelettique, le foie et l'intestin. L'intestin pourrait avoir un rôle prépondérant puisqu'il constitue l'organe immunitaire le plus large de l'organisme et qu'il comporte des milliards de bactéries qui peuvent relarguer des stimuli inflammatoires dans la circulation. En dépit de son importance, les mécanismes fins (cellulaires et moléculaires) conduisant à ces sécrétions chroniques de bas bruit ne sont pas élucidés mais plusieurs hypothèses ont été émises incluant le stress, le stress oxydatif [31], les lésions persistantes de l'ADN [32], le vieillissement des cellules souches [33] et l'inhibition de l'autophagie par l'activation de l'inflammasome [34]. Cette exposition continue à des débris cellulaires, des virus, des microbes ou des radiations induit la sénescence cellulaire, associée au SASP (proinflammatory senescence-associated secretory phenotype). Tous ces mécanismes sont probablement intriqués et conduisent à la production de résidu du stress oxydatif (ROS). Les ROS entrainent des dommages cellulaires oxydatifs et amplifient la sécrétion de cytokines pro-inflammatoires, créant un véritable cercle vicieux où les lésions tissulaires et les 
mécanismes de cicatrisation progressent parallèlement, tandis que les dommages

s'accumulent lentement et asymptomatiquement à travers les décennies. De plus, les altérations endocriniennes et métaboliques sont liées au déplacement de la production de cytokines vers un profil pro-inflammatoire, ce qui pourrait expliquer certaines pathologies liées à l'âge (maladie d'Alzheimer, maladie de Parkinson, pathologies cardio-vasculaires, ostéoporose, diabète, cancer et fragilité) [35,36].

A contrario, les patients âgés présentant des taux de cytokines anti-inflammatoires élevé semblent vieillir en «meilleure santé » que les autres. Ainsi, un taux élevé d'IL-10 est associé à un moindre risque de mortalité cardio-vasculaire [37] et se retrouve plus fréquemment chez les centenaires [38]. Le TGF- $\beta$ se trouve également en concentration supérieure chez les centenaires comparativement aux sujets jeunes [38,39].

\section{Impact du stress sur le système immunitaire}

Le stress peut se définir comme une réponse physiologique de l'organisme à une agression quelle qu'elle soit. Cette réponse peut être transitoire si l'agression est de courte durée (stress aigu) ou prolongée (stress chronique).

Les systèmes nerveux, endocrinien et immunitaire interagissent entre eux pour répondre de manière adaptée et efficace au stress [40]. L'axe hypothalamo-hypophyso-surrénalien est la principale voie par laquelle interagissent les 3 systèmes. En effet, lorsque l'organisme est soumis à un stress, l'hypothalamus induit la production d'" adrenocorticotropin hormone » (ACTH), d'hormone de croissance et de prolactine par l'hypophyse. L'ACTH stimule la glande surrénale entrainant la production de catécholamines (adrénaline, noradrénaline) et de glucocorticoïdes, autrement appelées «hormones de stress ». Ces hormones vont pouvoir se fixer sur les récepteurs de surface de différents types cellulaires (leucocytes, monocytes, macrophages), et ainsi moduler leur activité $[40,41]$. II existe peu de données à l'heure actuelle mais il semblerait qu'à la phase précoce (dans les premières heures) le stress induise une réponse pro-inflammatoire alors qu'à plus long terme cette réponse serait 
plutôt anti-inflammatoire. De plus, il existe un phénomène de rétrocontrôle par lequel le système immunitaire peut influencer l'axe hypothalamo-hypophysaire. En effet, la production d'IL-1 entraine une inhibition de la production de « corticotropine-releasing hormone » $(\mathrm{CRH})$ par l'hypothalamus et donc d'ACTH par l'hypophyse [40]. Par conséquent, le dérèglement de l'interaction entre le système nerveux et endocrinien peut avoir un impact sur les réponses immunitaires.

De nombreux événements cliniques peuvent être considérés comme des facteurs de stress aigus tels que la sepsis ou la fracture de l'extrémité supérieure du fémur. Ces événements, fréquents chez le sujet âgé, sont associés à un mauvais pronostic vital et fonctionnel.

\section{Fracture de l'extrémité supérieure du fémur (FESF)}

A travers le monde, 1,6 million de patients souffrent d'une FESF chaque année [42]. Cette pathologie touche principalement les sujets âgés, $80 \%$ des patients ayant plus de 75 ans [43]. Elles sont associées à un mauvais pronostic, avec 30\% de décès à 1 an et une perte d'autonomie fonctionnelle touchant près de la moitié des survivants [43]. De multiples facteurs tels que la chute, la fracture elle-même, la douleur et la chirurgie contribuent à considérer la FESF comme un bon modèle de stress aigu. Plusieurs études mettent en évidence des modifications du système immunitaire après une FESF, suggérant un rôle important de l'immunité dans l'évolution des patients. Les neutrophiles subissent une diminution transitoire de leur fonction de chimiotactisme et de phagocytose ainsi qu'une diminution de l'expression de CD16, une activation des voies de signalisation NFKB et PI3K et une inhibition de la NADPH oxydase pouvant impacter l'évolution clinique des patients [44]. De plus, les 3 sous-types de monocytes (classiques, intermédiaires et non conventionnels) subissent plusieurs altérations de leurs phénotypes et de leurs fonctions, notamment des monocytes non conventionnels. Leur fonction phagocytaire et leur production de superoxyde sont altérées, leur production de TNF- $\alpha$ augmente et leur production d'IL-10 diminue par rapport aux contrôles sains [44]. Ces résultats suggèrent un état pro-inflammatoire intense et transitoire après une fracture de la hanche. L'importance de 
cette inflammation, reflétée par les taux de cytokines pro-inflammatoire (IL-6, TNF-a, IL-10) et la procalcitonine, est associée au pronostic des patients et notamment à mortalité à long terme $[45,46]$. De plus, des taux plus élevés d'IL-6 et d'IL-1 $\beta$ sont retrouvés chez les patients présentant une confusion post-FESF comparativement aux contrôles $[47,48]$. Une publication récente a montré que les taux plasmatiques de néoptérine, une molécule

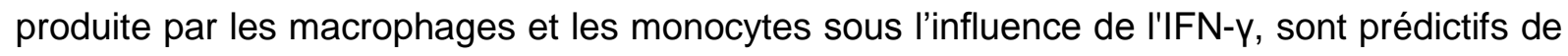
mortalité à un an d'une FESF chez les personnes âgées. De plus, la néoptérine, mesurée à l'arrivée à l'hôpital, est corrélée négativement au temps de survie après la chirurgie [49]. II est intéressant de noter que la voie conduisant à la production de néoptérine est couramment utilisée pour la synthèse de la 5,6,7,8-tétrahydrobioptérine (BH4), essentielle à la synthèse de la dopamine et de la sérotonine, deux hormones impliquées dans les réponses au stress.

\section{Le sepsis}

Le sepsis est treize fois plus fréquent dans la population âgée et est associé à deux fois plus de mortalité par rapport à la population jeune [50]. Deux mécanismes immunitaires sont décrits dans le sepsis : un premier stade pro-inflammatoire où une tempête de cytokines est observé puis une seconde phase immunosuppressive permettant le retour à l'équilibre homéostatique [51]. Des altérations immunitaires innées et adaptatives surviennent au cours du stade immunosuppresseur incluant l'altération fonctionnelle des monocytes, l'augmentation des lymphocytes T régulateurs, l'augmentation de l'expression immunitaire des inhibiteurs et la diminution de l'expression immunitaire sur les cellules T, l'augmentation des cytokines suppressives telles que l'IL-10 et le TGF- $\beta$ [51,52]. La persistance de ce stade suppressif est associée à un mauvais pronostic, principalement la mort et les infections secondaires. Plusieurs études précliniques ont testé des agents immunomodulateurs tels que l'interleukine 7 ou l'anti-PD1 sur des modèles de souris septiques, avec une amélioration significative de la survie [53,54]. Cependant, l'impact du sepsis sur le système immunitaire sénescent humain est encore mal compris et nécessite d'être approfondi. 


\section{Interventions cliniques pour un vieillissement en bonne santé}

Un modèle intéressant de vieillissement réussi est celui des centenaires, dont l'étude des facteurs associés à cette longévité pourrait être une piste dans la recherche de thérapeutiques ciblées. En 2008, une étude menée sur une cohorte de centenaires a montré la présence de variants génétiques dans la séquence codante de l'IGF-1R conduisant à une voie de signalisation IGF défectueuse, impliquée dans leur longévité [55]. Une autre étude a confirmé l'apparition de polymorphismes dans le gène $\mathrm{FOXO3a} \mathrm{associés} \mathrm{à} \mathrm{la} \mathrm{longévité} \mathrm{des}$ patients [56]. Récemment, en 2016, en réalisant le génotype des "single-nucleotide polymorphism » (SNP), les auteurs ont identifié des polymorphismes SIRT6 associés à la longévité humaine [57]. L'ensemble du séquençage des exons a permis d'améliorer la caractérisation et l'identification des gènes associés à la longévité en bonne santé [58]. Hormis leurs spécificités génétiques, les centenaires présentent un phénotype immunitaire particulier. La cohorte des centenaires italiens a révélé un profil immunitaire hautement conservé (nombre plus élevé de cellules T naïves et de cellules mémoire fonctionnelles avec une diversité de répertoires de lymphocytes $T$ conservée). Leur nombre de cellules B est maintenu avec un titre d'IgM accru, ce qui suggère une meilleure capacité de réponse à une nouvelle stimulation antigénique. De plus, leur activité métabolique est favorable avec une voie de l'insuline efficace [59] et une résistance au stress oxydatif [60], maintenant un équilibre entre l'environnement pro et anti-inflammatoire [60].

De nos jours, il n'existe pas d'intervention médicale ciblant le vieillissement faute de biomarqueurs spécifiques [35]. Parmi les pistes potentielles d'interventions anti-âge, la restriction calorique $(\mathrm{RC})$ a été proposée en raison de son effet bénéfique sur le stress oxydatif et de son effet anti-inflammatoire. Dans les modèles animaux, plusieurs études ont conclu à un effet positif de la RC sur la mortalité, l'autonomie fonctionnelle et plusieurs maladies comme la maladie d'Alzheimer, la maladie de Parkinson ou la cardiomyopathie [61]. Un essai clinique (étude CALERIE) a été conduit sur 220 adultes en bonne santé afin d'évaluer l'impact de la RC sur le vieillissement chez l'homme. Un effet positif de la RC est 
retrouvé sur 2 algorithmes biologiques de vieillissement [62]. Cependant, ces résultats sont clinique réalisé sur des sujets en bonne santé. De plus, la dénutrition qui est fréquente chez les personnes âgées, est connue pour être associée à la mortalité et à la fragilité [63,64]. Les études interventionnelles ciblant le processus de vieillissement humain et les maladies liées à l'âge sont en constante augmentation (résumées par Longo VD. et al. [65]). Ces stratégies, basées sur des études menées majoritairement chez les rongeurs, chez la levure Saccaromyces cerevisiae ou chez le nématode C. Elegans, visent principalement :

a) l'alimentation afin de limiter l'apport calorique (jeûne) ou l'apport protéique

b) l'inhibition de l'axe GH/ IGF-I (2-deoxy-D-glucose ; pegvisomant)

c) l'inhibition de la voie mTOR-S6K (rapamycine)

d) l'activation de la voie AMPK et des sirtuines (flavones, anthocyanines, metformine)

Chez l'homme, il existe peu d'essais cliniques ayant évalué l'efficacité de ces traitements (Tableau 1). Nous pouvons énumérer de manière non exhaustive, l'utilisation de la rapamycine (agent pharmacologique connu utilisé en transplantation et en oncologie), qui inhibe spécifiquement mTOR en créant un complexe tri-moléculaire entre mTOR kinase et la proteine FKBP. Son utilisation a conduit à l'amélioration des réponses vaccinales vis-à-vis du virus de la grippe [66]. En revanche, elle n'a pas d'effet significatif sur l'état cognitif et fonctionnel des patients [67]. De plus, la rapamycine a de nombreux effets indésirables, notamment de dérégulation métabolique (hyperglycémie, hyperinsulinemie, résistance à l'insuline [68]), ce qui limite son déploiement comme thérapie anti-âge.

Dans d'autres essais, l'utilisation de la metformine chez les adultes à haut risque de diabète de type 2 montre une incidence réduite de facteurs de risques cardio-vasculaires [69] et de maladies liées à l'âge tel que les cancers [70] et le déclin cognitif [71]. Sur la base de ces observations, la FDA vient d'approuver que la metformine puisse être testée sur la santé globale chez les personnes âgées et la première étude en cours nommée TAME 
(vieillissement ciblé avec la metformine) pourrait être le premier pas vers le développement de médicaments anti-âge efficaces.

En outre, certaines interventions nutritionnelles ont été suggérées comme des agents antiinflammatoires prometteurs et pourraient même jouer sur les réponses inflammatoires au stress $[72,73]$.

A ce jour, de nombreuses pistes thérapeutiques sont proposées dans la littérature scientifique, avec des résultats très encourageants dans les modèles animaux. II est encore impossible de conclure sur l'efficacité de ces thérapeutiques chez l'homme en raison du faible nombre d'essais cliniques publié.

\section{Conclusion}

Le vieillissement physiologique est associé à des changements du système neuroendocrinien et à des altérations du système immunitaire au sein des compartiments innés et adaptatifs. De nombreux liens existent entre le stress et l'immunité, conduisant à un niveau élevé d'inflammation, nécessaire pour défendre son intégrité mais qui doit être contrôlé afin de conserver un équilibre immunitaire. Les personnes âgées ne sont pas égales face au stress, notamment de par la grande variabilité interindividuelle en termes de réserve immunitaire. Une meilleure compréhension des interactions entre stress et immunité pourrait permettre d'appréhender les mécanismes impliqués dans l'impact du système immunitaire sur la résilience après un stress aigu chez les personnes âgées mais aussi d'envisager des thérapeutiques ciblées spécifiques des altérations du système immunitaire afin d'améliorer le pronostic des patients. A terme, l'immunomodulation pourrait être une approche thérapeutique intéressante chez le sujet âgé soumis à un stress aigu mais l'équilibre bénéfice / risque doit d'abord être évalué chez cette population particulièrement immunosénescente. 


\section{Remerciements}

Les auteurs sont reconnaissants à la Société Française de Gériatrie et de Gérontologie pour l'obtention du prix Chaffoteaux attribué par la Fondation de France à Hélène Vallet.

\section{Conflits d'intérêts}

Les auteurs ne déclarent aucun conflit d'intérêt. 


\section{Références}

1. Population Division Department of Economic and Social Affairs. World Population Ageing: 1950-2050 [Internet]. 2001. Disponible sur:

http://www.un.org/esa/population/publications/worldageing19502050/index.htm

2. Bouchon JP. 1+2+3 ou comment tenter d'être efficace en gériatrie? Rev Prat. 1984;34:888- 92.

3. López-Otín C, Blasco MA, Partridge L, Serrano M, Kroemer G. The hallmarks of aging. Cell.2013;153(6):1194- 217.

4. El Assar M, Angulo J, Carnicero JA, Walter S, García-García FJ, López-Hernández E, et al. Frailty Is Associated With Lower Expression of Genes Involved in Cellular Response to Stress: Results From the Toledo Study for Healthy Aging. J Am Med Dir Assoc.2017;18(8):734.e1- 734.e7.

5. Goronzy JJ, Weyand CM. Understanding immunosenescence to improve responses to vaccines. Nat Immunol.2013;14(5):428- 36.

6. Nyugen J, Agrawal S, Gollapudi S, Gupta S. Impaired functions of peripheral blood monocyte subpopulations in aged humans. J Clin Immunol.2010;30(6):806- 13.

7. Hearps AC, Martin GE, Angelovich TA, Cheng W-J, Maisa A, Landay AL, et al. Aging is associated with chronic innate immune activation and dysregulation of monocyte phenotype and function. Aging Cell. 2012;11(5):867- 75.

8. De Martinis M, Modesti M, Ginaldi L. Phenotypic and functional changes of circulating monocytes and polymorphonuclear leucocytes from elderly persons. Immunol Cell Biol.2004;82(4):415- 20.

9. Fortin CF, McDonald PP, Lesur O, Fülöp T. Aging and neutrophils: there is still much to do. Rejuvenation Res.2008;11(5):873- 82.

10. Angelis $\mathrm{P}$, Scharf $\mathrm{S}$, Christophidis $\mathrm{N}$. Effects of age on neutrophil function and its relevance to bacterial infections in the elderly. J Clin Lab Immunol.1997;49(1):33- 40.

11. Corberand J, Ngyen F, Laharrague P, Fontanilles AM, Gleyzes B, Gyrard E, et al. Polymorphonuclear functions and aging in humans. J Am Geriatr Soc. 1981;29(9):391- 7.

12. Sauce D, Dong Y, Campillo-Gimenez L, Casulli S, Bayard C, Autran B, et al. Reduced Oxidative Burst by Primed Neutrophils in the Elderly Individuals Is Associated With Increased Levels of the CD16bright/CD62Ldim Immunosuppressive Subset. J Gerontol A Biol Sci Med Sci.2017;72(2):163- 72.

13. Butcher SK, Chahal H, Nayak L, Sinclair A, Henriquez NV, Sapey E, et al. Senescence in innate immune responses: reduced neutrophil phagocytic capacity and CD16 expression in elderly humans. J Leukoc Biol.2001;70(6):881- 6.

14. Fulop T, Larbi A, Douziech N, Fortin C, Guérard K-P, Lesur O, et al. Signal transduction and functional changes in neutrophils with aging. Aging Cell. 2004;3(4):217- 26.

15. Sauce D, Larsen M, Fastenackels S, Duperrier A, Keller M, Grubeck-Loebenstein B, et al. Evidence of premature immune aging in patients thymectomized during early childhood. J Clin Invest. 2009;119(10):3070- 8. 
16. Zlamy M, Almanzar G, Parson W, Schmidt C, Leierer J, Weinberger B, et al. Efforts of the human immune system to maintain the peripheral CD8+ T cell compartment after childhood thymectomy. Immun Ageing. 2016;13:3.

17. Naylor K, Li G, Vallejo AN, Lee W-W, Koetz K, Bryl E, et al. The influence of age on T cell generation and TCR diversity. J Immunol Baltim Md 1950. 2005;174(11):7446- 52.

18. Britanova OV, Putintseva EV, Shugay M, Merzlyak EM, Turchaninova MA, Staroverov DB, et al. Age-related decrease in TCR repertoire diversity measured with deep and normalized sequence profiling. J Immunol Baltim Md 1950. 2014;192(6):2689- 98.

19. Sansoni P, Vescovini R, Fagnoni F, Biasini C, Zanni F, Zanlari L, et al. The immune system in extreme longevity. Exp Gerontol.2008;43(2):61- 5.

20. Tarazona R, DelaRosa O, Alonso C, Ostos B, Espejo J, Peña J, et al. Increased expression of NK cell markers on T lymphocytes in aging and chronic activation of the immune system reflects the accumulation of effector/senescent T cells. Mech Ageing Dev. 2000;121(1-3):77- 88.

21. Nikolich-Zugich J. Ageing and life-long maintenance of T-cell subsets in the face of latent persistent infections. Nat Rev Immunol. 2008;8(7):512- 22.

22. Fann M, Chiu WK, Wood WH, Levine BL, Becker KG, Weng N-P. Gene expression characteristics of CD28null memory phenotype CD8+ T cells and its implication in T-cell aging. Immunol Rev.2005;205:190- 206.

23. Raynor J, Lages CS, Shehata H, Hildeman DA, Chougnet CA. Homeostasis and function of regulatory T cells in aging. Curr Opin Immunol. 2012;24(4):482- 7.

24. Hwang K-A, Kim H-R, Kang I. Aging and human CD4(+) regulatory T cells. Mech Ageing Dev.2009;130(8):509- 17.

25. Frasca D, Diaz A, Romero M, Blomberg BB. Human peripheral late/exhausted memory $B$ cells express a senescent-associated secretory phenotype and preferentially utilize metabolic signaling pathways. Exp Gerontol. 2017;87(Pt A):113- 20.

26. Johnson SA, Cambier JC. Ageing, autoimmunity and arthritis: senescence of the B cell compartment - implications for humoral immunity. Arthritis Res Ther. 2004;6(4):131- 9.

27. Naradikian MS, Hao Y, Cancro MP. Age-associated B cells: key mediators of both protective and autoreactive humoral responses. Immunol Rev. 2016;269(1):118- 29.

28. Franceschi C, Campisi J. Chronic inflammation (inflammaging) and its potential contribution to age-associated diseases. J Gerontol A Biol Sci Med Sci. 2014;69 Suppl 1:S4- 9.

29. Fagiolo U, Cossarizza A, Scala E, Fanales-Belasio E, Ortolani C, Cozzi E, et al. Increased cytokine production in mononuclear cells of healthy elderly people. Eur J Immunol.1993;23(9):2375- 8.

30. Franceschi C, Capri M, Monti D, Giunta S, Olivieri F, Sevini F, et al. Inflammaging and antiinflammaging: a systemic perspective on aging and longevity emerged from studies in humans. Mech Ageing Dev. 2007;128(1):92- 105.

31. Cannizzo ES, Clement CC, Sahu R, Follo C, Santambrogio L. Oxidative stress, inflamm-aging and immunosenescence. J Proteomics. 2011;74(11):2313- 23. 
32. Olivieri F, Albertini MC, Orciani M, Ceka A, Cricca M, Procopio AD, et al. DNA damage response (DDR) and senescence: shuttled inflamma-miRNAs on the stage of inflamm-aging. Oncotarget. 2015;6(34):35509- 21.

33. Jones DL, Rando TA. Emerging models and paradigms for stem cell ageing. Nat Cell Biol. 2011;13(5):506- 12.

34. Salminen A, Kaarniranta K, Kauppinen A. Inflammaging: disturbed interplay between autophagy and inflammasomes. Aging. 2012;4(3):166- 75.

35. Xia S, Zhang X, Zheng S, Khanabdali R, Kalionis B, Wu J, et al. An Update on Inflamm-Aging: Mechanisms, Prevention, and Treatment. J Immunol Res. 2016;2016:8426874.

36. Fülöp T, Dupuis G, Witkowski JM, Larbi A. The Role of Immunosenescence in the Development of Age-Related Diseases. Rev Investig Clin Organo Hosp Enfermedades Nutr. 2016;68(2):84- 91.

37. Van Den Biggelaar AHJ, De Craen AJM, Gussekloo J, Huizinga TWJ, Heijmans BT, Frölich M, et al. Inflammation underlying cardiovascular mortality is a late consequence of evolutionary programming. FASEB J Off Publ Fed Am Soc Exp Biol. 2004;18(9):1022- 4.

38. Salvioli S, Capri M, Bucci L, Lanni C, Racchi M, Uberti D, et al. Why do centenarians escape or postpone cancer? The role of IGF-1, inflammation and p53. Cancer Immunol Immunother Cll.2009;58(12):1909- 17.

39. Carrieri G, Marzi E, Olivieri F, Marchegiani F, Cavallone L, Cardelli M, et al. The G/C915 polymorphism of transforming growth factor beta1 is associated with human longevity: a study in Italian centenarians. Aging Cell. 2004;3(6):443- 8.

40. Glaser R, Kiecolt-Glaser JK. Stress-induced immune dysfunction: implications for health. Nat Rev Immunol. 2005;5(3):243- 51.

41. Padgett DA, Glaser R. How stress influences the immune response. Trends Immunol. 2003;24(8):444- 8.

42. Borgström F, Lekander I, Ivergård M, Ström O, Svedbom A, Alekna V, et al. The International Costs and Utilities Related to Osteoporotic Fractures Study (ICUROS)--quality of life during the first 4 months after fracture. Osteoporos Int J Establ Result Coop Eur Found Osteoporos Natl Osteoporos Found USA.2013;24(3):811- 23.

43. Brauer CA, Coca-Perraillon M, Cutler DM, Rosen AB. Incidence and mortality of hip fractures in the United States. JAMA J Am Med Assoc. 2009;302(14):1573- 9.

44. Baëhl S, Garneau H, Le Page A, Lorrain D, Viens I, Svotelis A, et al. Altered neutrophil functions in elderly patients during a 6-month follow-up period after a hip fracture. Exp Gerontol.2015;65:58- 68 .

45. Sun $T$, Wang $X$, Liu Z, Chen X, Zhang J. Plasma concentrations of pro- and anti-inflammatory cytokines and outcome prediction in elderly hip fracture patients. Injury. 2011;42(7):707- 13.

46. Vallet H, Chenevier-Gobeaux C, Villain C, Cohen-Bittan J, Ray P, Epelboin L, et al. Prognostic Value of Serum Procalcitonin After Orthopedic Surgery in the Elderly Population. J Gerontol A Biol Sci Med Sci. 2017;72(3):438- 43. 
47. Neerland BE, Hall RJ, Seljeflot I, Frihagen F, MacLullich AMJ, Raeder J, et al. Associations Between Delirium and Preoperative Cerebrospinal Fluid C-Reactive Protein, Interleukin-6, and Interleukin-6 Receptor in Individuals with Acute Hip Fracture. J Am Geriatr Soc.2016;64(7):1456- 63.

48. Cape E, Hall RJ, van Munster BC, de Vries A, Howie SEM, Pearson A, et al. Cerebrospinal fluid markers of neuroinflammation in delirium: a role for interleukin- $1 \beta$ in delirium after hip fracture. J Psychosom Res. 2014;77(3):219- 25.

49. Larsen M, Bayard C, Lepetitcorps H, Cohen-Bittan J, Appay V, Boddaert J, et al. Elevated Neopterin Levels Predict Early Death in Older Hip-fracture Patients.

EBioMedicine.2017;26:157- 64.

50. Martin GS, Mannino DM, Moss M. The effect of age on the development and outcome of adult sepsis. Crit Care Med. 2006;34(1):15- 21.

51. Delano MJ, Ward PA. The immune system's role in sepsis progression, resolution, and longterm outcome. Immunol Rev. 2016;274(1):330- 53.

52. Rittirsch D, Flierl MA, Ward PA. Harmful molecular mechanisms in sepsis. Nat Rev Immunol. 2008;8(10):776- 87.

53. Brahmamdam P, Inoue S, Unsinger J, Chang KC, McDunn JE, Hotchkiss RS. Delayed administration of anti-PD-1 antibody reverses immune dysfunction and improves survival during sepsis. J Leukoc Biol. 2010;88(2):233- 40.

54. Unsinger J, McGlynn M, Kasten KR, Hoekzema AS, Watanabe E, Muenzer JT, et al. IL-7 promotes T cell viability, trafficking, and functionality and improves survival in sepsis. J Immunol Baltim Md 1950. 2010;184(7):3768- 79.

55. Suh Y, Atzmon G, Cho M-O, Hwang D, Liu B, Leahy DJ, et al. Functionally significant insulin-like growth factor I receptor mutations in centenarians. Proc Natl Acad Sci U S A. 2008;105(9):3438- 42.

56. Flachsbart $F$, Caliebe $A$, Kleindorp $R$, Blanché $H$, von Eller-Eberstein $H$, Nikolaus $\mathrm{S}$, et al. Association of FOXO3A variation with human longevity confirmed in German centenarians. Proc Natl Acad Sci U S A.2009;106(8):2700- 5.

57. Li Y, Qin J, Wei X, Liang G, Shi L, Jiang M, et al. Association of SIRT6 Gene Polymorphisms with Human Longevity. Iran J Public Health. 2016;45(11):1420- 6.

58. Dewey FE, Murray MF, Overton JD, Habegger L, Leader JB, Fetterolf SN, et al. Distribution and clinical impact of functional variants in 50,726 whole-exome sequences from the DiscovEHR study. Science. 2016;354(6319).

59. Bucci L, Ostan R, Giampieri E, Cevenini E, Pini E, Scurti M, et al. Immune parameters identify Italian centenarians with a longer five-year survival independent of their health and functional status. Exp Gerontol. 2014;54:14- 20.

60. Franceschi C, Monti D, Sansoni P, Cossarizza A. The immunology of exceptional individuals: the lesson of centenarians. Immunol Today.1995;16(1):12- 6. 
61. Chung KW, Kim DH, Park MH, Choi YJ, Kim ND, Lee J, et al. Recent advances in calorie restriction research on aging. Exp Gerontol. 2013;48(10):1049- 53.

62. Belsky DW, Huffman KM, Pieper CF, Shalev I, Kraus WE. Change in the Rate of Biological Aging in Response to Caloric Restriction: CALERIE Biobank Analysis. J Gerontol A Biol Sci Med Sci.2017;73(1):4- 10.

63. Harris D, Haboubi N. Malnutrition screening in the elderly population. J R Soc Med.2005;98(9):411- 4.

64. Fried LP, Tangen CM, Walston J, Newman AB, Hirsch C, Gottdiener J, et al. Frailty in older adults: evidence for a phenotype. J Gerontol A Biol Sci Med Sci. 2001;56(3):M146- 56.

65. Longo VD, Antebi A, Bartke A, Barzilai N, Brown-Borg HM, Caruso C, et al. Interventions to Slow Aging in Humans: Are We Ready? Aging Cell. 2015;14(4):497- 510.

66. Mannick JB, Del Giudice G, Lattanzi M, Valiante NM, Praestgaard J, Huang B, et al. mTOR inhibition improves immune function in the elderly. Sci Transl Med. 2014;6(268):268ra179.

67. Kraig $\mathrm{E}$, Linehan $\mathrm{LA}$, Liang $\mathrm{H}$, Romo $\mathrm{TQ}$, Liu $\mathrm{Q}, \mathrm{Wu} \mathrm{Y}$, et al. A randomized control trial to establish the feasibility and safety of rapamycin treatment in an older human cohort: Immunological, physical performance, and cognitive effects. Exp Gerontol. 2018;105:53- 69.

68. Soefje SA, Karnad A, Brenner AJ. Common toxicities of mammalian target of rapamycin inhibitors. Target Oncol. 2011;6(2):125- 9.

69. Abbasi F, Chu JW, McLaughlin T, Lamendola C, Leary ET, Reaven GM. Effect of metformin treatment on multiple cardiovascular disease risk factors in patients with type 2 diabetes mellitus. Metabolism. 2004;53(2):159- 64.

70. Anisimov VN. Metformin for aging and cancer prevention. Aging. 2010;2(11):760- 74.

71. Ng TP, Feng L, Yap KB, Lee TS, Tan CH, Winblad B. Long-term metformin usage and cognitive function among older adults with diabetes. J Alzheimers Dis JAD. 2014;41(1):61- 8.

72. Maes $M$, Lin A, Kenis $G$, Egyed B, Bosmans $E$. The effects of noradrenaline and alpha-2 adrenoceptor agents on the production of monocytic products. Psychiatry Res.2000;96(3):245- 53.

73. Kiecolt-Glaser JK, Belury MA, Porter K, Beversdorf DQ, Lemeshow S, Glaser R. Depressive symptoms, omega-6:omega-3 fatty acids, and inflammation in older adults. Psychosom Med. 2007;69(3):217- 24.

74. Turner RS, Thomas RG, Craft S, van Dyck CH, Mintzer J, Reynolds BA, et al. A randomized, double-blind, placebo-controlled trial of resveratrol for Alzheimer disease. Neurology.2015;85(16):1383- 91.

75. Huhn S, Beyer F, Zhang R, Lampe L, Grothe J, Kratzsch J, et al. Effects of resveratrol on memory performance, hippocampus connectivity and microstructure in older adults - A randomized controlled trial. Neurolmage. 2018;174:177- 90. 
1

2

3

4

7

8

10

11

12

13

14

15

16

17

18

19

20

21

22

23

24

25

26

27

28

29

30

31

32

33

34

35

36

37

38

39

40

41

42

43

44

45

46

47

48

49

50

51

52

53

54

55

56

57

58

59

60

61

62

63

64

65 
Tableau 1 : Principaux essais cliniques et études chez l'homme portant sur l'impact des thérapeutiques anti-vieillissement

\begin{tabular}{|c|c|c|c|c|c|}
\hline & $\begin{array}{c}\text { Thérapeutique } \\
\text { utilisée }\end{array}$ & Mode d'action & Etude & Population étudiée & Principaux résultats \\
\hline $\begin{array}{l}\text { Mannick et al. } \\
2014[66]\end{array}$ & Rapamycine & $\begin{array}{l}\text { Inhibition de la } \\
\text { voie mTOR }\end{array}$ & $\begin{array}{l}\text { Randomisée contre } \\
\text { placebo }\end{array}$ & $\begin{array}{l}218 \text { patients, âge } \geq 65 \\
\text { ans }\end{array}$ & $\begin{array}{l}37 \% \text { d'effets secondaires } \\
\text { Amélioration des réponses vaccinales antigrippales, } \\
\text { réduction du \% de lymphocytes T exprimant PD-1 }\end{array}$ \\
\hline $\begin{array}{l}\text { Kraig et al. } 2018 \\
{[67]}\end{array}$ & Rapamycine & $\begin{array}{l}\text { Inhibition de la } \\
\text { voie mTOR }\end{array}$ & $\begin{array}{l}\text { Randomisée contre } \\
\text { placebo }\end{array}$ & $\begin{array}{l}25 \text { patients, moyenne } \\
\text { d'âge } 80 \text { ans }\end{array}$ & $\begin{array}{l}\text { Bonne tolérance } \\
\text { Pas de différence significative entre les } 2 \text { groupes à } 8 \\
\text { semaines en termes de profil immunologique (sous types } \\
\text { cellulaires et profil cytokinique) et de résultats cliniques (état } \\
\text { cognitif et fonctionnel) }\end{array}$ \\
\hline $\begin{array}{l}\text { Belsky et al. } 2017 \\
{[62]}\end{array}$ & $\begin{array}{l}\text { Restriction } \\
\text { calorique }\end{array}$ & & $\begin{array}{l}\text { Randomisée contre } 1 \\
\text { groupe contrôle sans } \\
\text { restriction calorique }\end{array}$ & $\begin{array}{l}220 \text { patients, moyenne } \\
\text { d'âge } 38 \text { ans }\end{array}$ & $\begin{array}{l}\text { Vieillissement biologique significativement ralenti dans le } \\
\text { groupe « restriction calorique » }\end{array}$ \\
\hline $\begin{array}{l}\text { Abbasi et al. } 2004 \\
{[69]}\end{array}$ & Metformine & $\begin{array}{l}\text { Activation de la } \\
\text { voie AMPK }\end{array}$ & Etude cas-témoin & $\begin{array}{l}31 \text { patients, diabète } \\
\text { type } 2 \text { sous régime } \\
\text { seul ou sulfamide en } \\
\text { monothérapie, } \\
\text { moyenne d'âge } 58 \text { ans }\end{array}$ & $\begin{array}{l}\text { Diminution significative du taux plasmatique de glucose, de } \\
\text { triglycéride et d'acide gras libre sous Metformine }\end{array}$ \\
\hline Ng et al. 2014 [71] & Metformine & $\begin{array}{l}\text { Activation de la } \\
\text { voie AMPK }\end{array}$ & Etude de cohorte & $\begin{array}{l}367 \text { patients } \\
\text { diabétiques, âge } \\
\text { moyen } 67 \text { ans }\end{array}$ & $\begin{array}{l}\text { Les patients traités par metformine étaient moins à risque de } \\
\text { développer des troubles cognitifs que les patients non traités. }\end{array}$ \\
\hline $\begin{array}{l}\text { Turner et al. } 2015 \\
{[74]}\end{array}$ & Resveratrol & $\begin{array}{l}\text { Activation de } \\
\text { SIRT1 (voie des } \\
\text { sirtuines) }\end{array}$ & $\begin{array}{l}\text { Randomisée contre } \\
\text { placebo }\end{array}$ & $\begin{array}{l}119 \text { patients atteints } \\
\text { de maladie } \\
\text { d'Alzheimer, âge } \\
\text { moyen } 72 \text { ans }\end{array}$ & $\begin{array}{l}\text { Bonne tolérance du traitement } \\
\text { Diminution plus importante de l'A } \beta \text { sanguine et dans le LCR } \\
\text { dans groupe placebo }\end{array}$ \\
\hline $\begin{array}{l}\text { Huhn et al. } 2018 \\
{[75]}\end{array}$ & Resveratrol & $\begin{array}{l}\text { Activation de } \\
\text { SIRT1 (voie des } \\
\text { sirtuines) }\end{array}$ & $\begin{array}{l}\text { Randomisée contre } \\
\text { placebo }\end{array}$ & $\begin{array}{l}60 \text { patients, âge } \\
\text { moyen } 67 \text { ans }\end{array}$ & $\begin{array}{l}\text { Pas d'amélioration significative de la mémoire sous } \\
\text { resveratrol }\end{array}$ \\
\hline
\end{tabular}


Facteurs intrinsèques

(hôte)
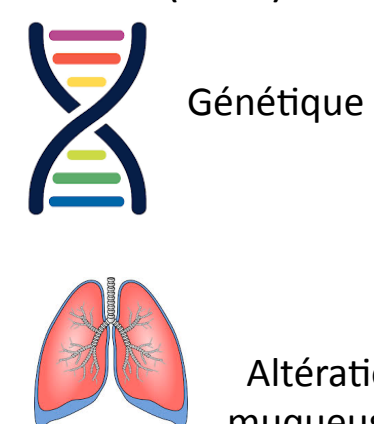

Altérations des muqueuses et du microbiote
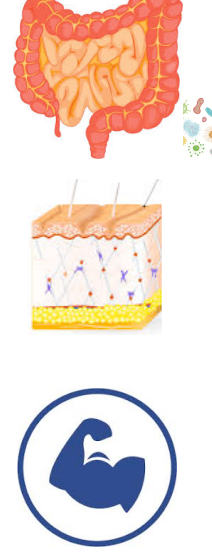

Atrophie de la peau

et de la masse

musculaire (sarcopénie)

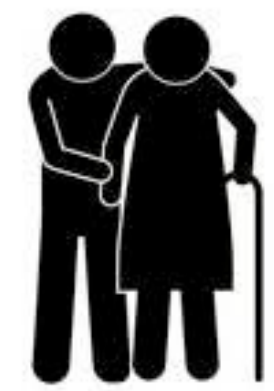

Vieillissement
Facteurs extrinsèques

(environnement)

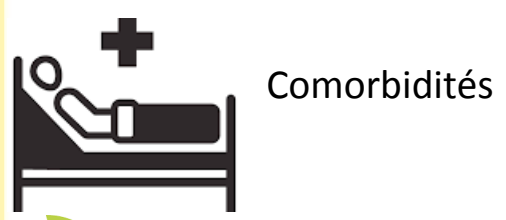

Régime alimentaire

Activité physique

Traitements
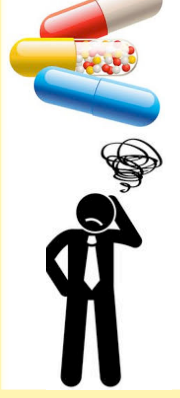

Stress, anxiété, dépression
Inflammation chronique

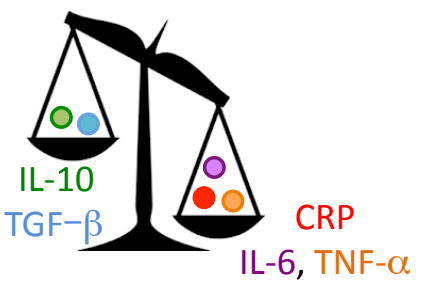

Changements des sous-populations lymphocytaires
Involution thymique

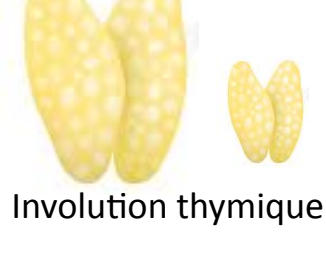

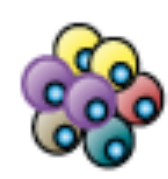

Moins de cellules naives
Accumulation de cellules

sénescentes

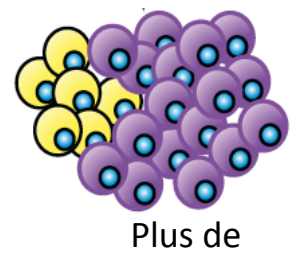

cellules mémoire

oligoclonales
Raccourcissement des télomères

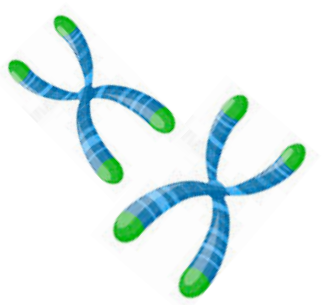

\section{Conséquences cliniques}

Pathologies auto-immunes, maladies cardio-vasculaires, cancers

Sévérité des infections

Efficacité des réponses vaccinales 